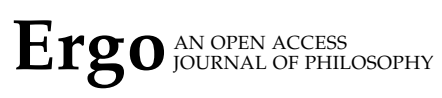

\title{
Obligation, Permission, AND BAYESIAN ORgulity
}

\author{
MICHAEL NIELSEN \\ Columbia University \\ RUSH T. STEWART \\ LMU Munich
}

\begin{abstract}
This essay has two aims. The first is to correct an increasingly popular way of misunderstanding Belot's (2013) Orgulity Argument. The Orgulity Argument charges Bayesianism with defect as a normative epistemology. For concreteness, our argument focuses on Cisewski et al.'s (2018) recent rejoinder to Belot. The conditions that underwrite their version of the argument are too strong and Belot does not endorse them on our reading. A more compelling version of the Orgulity Argument than Cisewski et al. present is available, however-a point that we make by drawing an analogy with de Finetti's (1974) argument against mandating countable additivity. Having presented the best version of the Orgulity Argument, our second aim is to develop a reply to it. We extend Elga's 2016) idea of appealing to finitely additive probability to show that the challenge posed by the Orgulity Argument can be met.
\end{abstract}

Keywords: Finite additivity; modesty; orgulity; probability; topology

\section{Introduction}

How much confidence does rationality demand in one's ability to get at the truth? A natural thought is that it depends on the nature of the inquiry at hand. A certain amount of humility might be understandable in the face of sufficiently complex problems. But, for Bayesians, such humility is out of the question. Coherence compels Bayesians to always be fully confident. Based on this fact, Gordon Belot (2013) advances an argument against Bayesianism that we call the Orgulity

Contact: Michael Nielsen <mn2683@columbia.edu>, Rush T.

Stewart <rush.stewart@lrz.uni-muenchen.de> 
Argument. The upshot of the Orgulity Argument is that Bayesianism-one of the most compelling, popular, powerful, and principled epistemological frameworks ever studied-is defective as a normative theory.

The argument has already proven to be influential and has produced a growing literature (Belot 2017; Cisewski et al. 2018; Elga 2016; Huttegger 2015a; Pomatto \& Sandroni 2018; Weatherson 2015). This literature raises several interesting and important points about the foundations of Bayesian learning. But we have found that many contributors to this literature have misread Belot and have failed to identify the best version of the Orgulity Argument. This is important because, if we are correct, the challenge posed to Bayesianism by the Orgulity Argument is not being addressed, let alone met by most contributions to this debate. In order for the debate to advance, it is necessary to correct these mistakes. That is the first aim of this essay. Below, we focus on the most recent contribution to the debate from Cisewski et al. (2018), though we point out that our argument applies to other readers of Belot.

We argue that Cisewski et al.'s version of the Orgulity Argument relies on two premises that Belot does not endorse and, interpretative issues aside, are too strong to underwrite a plausible challenge to Bayesianism. The main point is simple. There is a clear difference between mandating certain behavior and insisting that the behavior is permissible. On Cisewski et al.'s reading of the Orgulity Argument, a certain kind of epistemological modesty (to be explained below) is rationally mandatory. On our reading, the argument relies on a considerably weaker premise: such modesty is permissible.

Having articulated the correct way to understand the Orgulity Argument, the second aim of our essay is to defend a line of response to the argument, initiated by Elga (2016), that involves relaxing countable additivity. Here, the first part of the essay proves useful. The tools that we develop for thinking about the correct version of the Orgulity Argument also help us to see that criticisms of Elga's reply are flawed. We then extend Elga's argument, showing that replies to the Orgulity Argument based on finitely additive probability theory are surprisingly robust.

After introducing the framework and the relevant concepts for the debate in Section 2, we present and discuss the Orgulity Argument as Cisewski et al. understand it (Section 3). By our lights, their interpretation exemplifies a common way of misunderstanding Belot's point. We then present a better version of the Orgulity Argument and clarify the normative confusion at stake by drawing an analogy to de Finetti's (1974) argument against mandating countable additivity (Sections 4 and 5). Finally, in Section 6 , we develop the finitely additive approach to answering the Orgulity Argument. 


\section{Preliminaries}

All versions of the Orgulity Argument rely on the mathematical concepts that we introduce in this section. Let $\mathcal{C}=\{0,1\}^{\mathbb{N}}$ be the set of all countably infinite binary sequences. We could interpret $\mathcal{C}$ as the set of possible outcomes of an experiment in which a coin is tossed infinitely many times, for example. In order to produce a probability space, we pair $\mathcal{C}$ with a particular sigma-algebra of its subsets, which we denote by $\mathscr{B} .{ }^{1}$ Elements of $\mathscr{B}$ are called hypotheses or events.

Bayesianism is the view that a rational agent's state of uncertainty regarding some set of live possibilities is represented by a probability measure. ${ }^{2}$ This probability measure is subject to revision as the agent learns. In particular, agents learn by conditionalization, forming posterior probabilities from prior conditional probabilities. The Orgulity Argument is a critique of the epistemological significance often attributed to the Bayesian convergence-to-the-truth theorem. To present the theorem, we introduce some more notation. Let $P$ be a probability measure on $(\mathcal{C}, \mathscr{B})$. If $\omega \in \mathcal{C}$, let $\omega^{n}$ be the set of sequences that agree with $\omega$ up to the $n^{\text {th }}$ digit. If $H$ is an event in $\mathscr{B}$, let $\mathbf{1}_{H}$ be the indicator function for $H .{ }^{3}$ The convergence-to-the-truth theorem says that

$$
P\left(\left\{\omega: \mathbf{1}_{H}(\omega)=\lim _{n \rightarrow \infty} P\left(H \mid \omega^{n}\right)\right\}\right)=1
$$

for all hypotheses H. ${ }^{4}$ Suppose that $\omega$ is the actual sequence of 0 s and $1 \mathrm{~s}$, and that this sequence is to be revealed one bit at a time to an agent with personal probabilities given by $P$. After observing the $n^{\text {th }}$ bit, the agent updates her probabilities by conditionalizing on $\omega^{n}$. Then, (1) says that for any hypothesis $H$, the agent believes (with probability 1 ) that her posterior probabilities for $H$ will converge to 1 or 0 according to whether $H$ is true or false, respectively. For any hypothesis $H$, the agent believes that she will converge to the truth about $H$. We call the complement of the set that appears in $(\mathbf{I})$, namely $\left\{\omega: \mathbf{1}_{H}(\omega) \neq\right.$ $\left.\lim _{n \rightarrow \infty} P\left(H \mid \omega^{n}\right)\right\}$, the failure set for $H$ (with respect to $P$ ). If $\omega$ is in the failure set for $H$, then conditionalizing on $\omega^{n}$ will not lead $P$ to the truth about $H$. Another way of summarizing (I) is to say that, for any hypothesis $H$, a Bayesian agent assigns probability 0 to the failure set for $H$.

1. We endow $\mathcal{C}$ with the infinite product topology of discrete topologies on $\{0,1\}$. The resulting topological space is called Cantor space. $\mathscr{B}$ is the (Borel) sigma-algebra that is generated by the open subsets of Cantor space.

2. With Belot, we will assume for the moment that probabilities are countably additive. The probability $P$ satisfies countable additivity if, for any countable collection $\left\{A_{i}\right\}_{i \in \mathbb{N}}$ of pairwise disjoint hypotheses in $\mathscr{B}$, we have $P\left(\bigcup_{i=1}^{\infty} A_{i}\right)=\sum_{i=1}^{\infty} P\left(A_{i}\right)$. We return to countable additivity below.

3. $\mathbf{1}_{H}(\omega)=\left\{\begin{array}{l}1, \omega \in H \\ 0, \omega \notin H\end{array}\right.$

4. See, for example, Billingsley (2008. Theorem 35.6). 
The failure set for $H$ is probabilistically "negligible" or "small" because it is assigned probability 0 . The Orgulity Argument appeals to a distinct notion of smallness that uses topological concepts. A subset $A$ of a topological space is nowhere dense if its closure has empty interior, that is, the smallest closed set containing $A$ does not contain any open sets. Nowhere dense sets are topologically small. For example, any finite subset of $\mathbb{R}$ is nowhere dense in the standard topology. A set is called meager if it is the countable union of nowhere dense sets. ${ }^{5}$ Since countable unions of probability 0 sets have probability 0 , it makes sense to say that countable unions of probabilistically small sets are probabilistically small. Similarly, meager sets, like the nowhere dense sets that compose them, are often said to be small or "atypical." The complement of a meager set is a comeager (or residual) set and is topologically "large" or "typical."

We can now state the mathematical fact that is the driving force behind the Orgulity Argument. Topological and probabilistic notions of smallness need not coincide. In particular, there are probabilities $P$ on $(\mathcal{C}, \mathscr{B})$ and hypotheses $H$ in $\mathscr{B}$ such that the failure set for $H$ with respect to $P$ is comeager. ${ }^{6}$ But applying (I) to the failure set for $H$, we see that it is assigned probability 0 . Although the failure set for $H$ is topologically large, it is probabilistically small. An agent with personal probabilities given by $P$ assigns probability 0 to failing to converge to the truth about $H$ even though that event is "typical."

\section{Cisewski et al.'s Version of the Orgulity Argument}

As Cisewski et al. (2018) read Belot, assigning probability 0 to a topologically large set is a sign of epistemological immodesty. An agent with such probabilities is "practically certain" that a (topologically) typical event will not occur. The main premise of the Orgulity Argument, on Cisewski et al.'s reading, is that epistemological immodesty in this sense is rationally forbidden. More precisely, Cisewski et al. claim that the following conditions underwrite the Orgulity Argument.

Topological Condition \# $\mathbf{1}$ Do not assign probability 1 to a meager set.

Topological Condition \#2 Assign probability 0 to each hypothesis that is a meager set. ${ }^{7}$

5. Some further examples: the Cantor set is an uncountable set but is nowhere dense, and so meager. $Q$ is a countable and dense subset of $\mathbb{R}$, but is also meager.

6. Belot shows that if a probability is "open-minded" in a technical sense 2013 496), then its failure set for a hypothesis that is countable and dense is comeager 2013 499). Treating this sense of open-mindedness will not be essential for our purposes since we can focus directly on the existence of comeager failure sets.

7. Cisewski et al. qualify these conditions to apply only to meager sets of observables. It isn't entirely clear what they intend with this qualification. One possibility is that it indicates 
We will focus on Condition \#1, but our criticisms carry over straightforwardly to the "more demanding" Condition \#2.

As pointed out in the previous section, there exist probability spaces $(\mathcal{C}, \mathscr{B}, P)$ and meager subsets of $\mathcal{C}$ that are assigned $P$-probability 1 . Such a $P$ violates Topological Condition \#1. The conclusion of the Orgulity Argument, on Cisewski et al.'s reading, is that Bayesianism should be rejected as a theory of rationality. Cisewski et al. deny the Orgulity Argument's conclusion and go on to argue convincingly that the above topological conditions have some untenable consequences.

We agree with Cisewski et al. that the conclusion of the Orgulity Argument is that Bayesianism should be rejected but dispute the claim that the argument relies on either of the above topological conditions. Other commentators on the Orgulity Argument are not as explicit about the argument's premises as Cisewski et al., but there are several remarks in the literature that are suggestive of Topological Condition \#1. For example, according to Huttegger, Belot's argument "appears to be along the lines of 'if a set is residual in the topology, then it should have positive probability'" (2015b: 594). And similarly, Weatherson presents the Orgulity Argument as introducing the "requirement" of modesty for residual sets (2015 532). For Weatherson, a probability $P$ is modest with respect to a hypothesis $H$ if $P$ assigns positive probability to the failure set for $H .{ }^{8}$ To say that modesty is required for residual sets, then, seems tantamount to an endorsement of Topological Condition \#1.

As we interpret Belot, the above topological conditions do not in fact occur as premises in the Orgulity Argument. Belot ends his paper with the following summary: "The truth concerning Bayesian convergence-to-the-truth results is significantly worse than has been generally allowed-they constitute a real liability for Bayesianism by forbidding a reasonable epistemological modesty" (2013, emphasis ours). His indictment of Bayesianism is based on the claim that modest probability judgments, which ought to be permissible, are deemed impermissible (forbidden) on the Bayesian account. Cisewski et al. reply instead to a version of the Orgulity Argument according to which such modesty is mandatory. But the Orgulity Argument need not (and it appears that Belot does not) mandate modesty to arrive at its conclusion. We develop this alternative way to construe the Orgulity

that the above conditions do not apply to hypotheses whose truth values are not determined by (even infinitely many) observations. Bayesian convergence to the truth, at any rate, does not hold for such hypotheses (Huttegger 2015b 590). (Our claim in the introduction that coherence compels Bayesians to "always be fully confident" likewise fails for such hypotheses.) If this is what Cisewski et al. intend, then our omission is harmless since we have restricted ourselves to the space of observables.

8. We agree with Weatherson that this is the correct way to understand modesty in the context of the Orgulity Argument (cf. Section 5). 
Argument in Sections 4 and 5

But it is worth pointing out already that the Cisewski et al. topological conditions are not even plausible on their face. Consider the probability according to which each digit in $\omega$ is determined by the outcome of a fair coin toss. By the strong law of large numbers, the hypothesis that the limiting relative frequency of $1 \mathrm{~s}$ is $1 / 2$ is assigned probability 1 . But, it turns out, this hypothesis is a meager subset of $\mathcal{C} .{ }^{9}$ Topological Condition \#1 implies that this probability measure is rationally impermissible. If one thinks that there are some contexts in which it is rationally permissible to adopt probabilities represented by the fair coin measure, then one should reject Topological Condition \#1. Whether or not Belot in fact endorses the topological conditions that Cisewski et al. attribute to him, a better version of the Orgulity Argument is available. This form of the argument relies on neither of the above topological conditions. We turn to this version of the Orgulity Argument now.

\section{An Analogy: de Finetti's Lottery}

We understand the Orgulity Argument in analogy to de Finetti's criticisms of countable additivity. Since we expect the latter to be familiar to most participants in the orgulity debate, we find it helpful to point out that the Orgulity Argument has the same structure as the argument based on de Finetti's lottery.

So far, we have assumed that all rational probability judgments are countably additive, but de Finetti denies this. On his view, if we take countable additivity to be a universal constraint on rational probability judgments, eminently reasonable credal states are ruled out. De Finetti does not insist that personal probability must always violate countable additivity. Rather, he insists that violating countable additivity is permissible (at least under certain circumstances). Insistence on countable additivity is heavy-handedness.

Consider a lottery consisting of countably many tickets, one for each integer. According to de Finetti, it should be open to a rational agent to consider such a lottery fair (de Finetti 1974). To do so, each ticket must be assigned equal probability. But if probabilities are countably additive, this is not possible. If each ticket is assigned the same positive probability, then the probability of the union of the tickets, being equal to the sum of the probabilities of the individual tickets, is infinite, and not 1 as the probability axioms demand. If, on the other hand, each ticket is assigned probability 0 , then so is the probability of their union. Again, this contradicts the probability axioms.

The example is meant to show that countable additivity is heavy-handed as 2013 85).

9. Belot suggested this example to us in personal communication. For details, see Oxtoby 
a constraint on rational probabilities. It forbids an opinion that is reasonable, at least in some circumstances, namely that a lottery on the integers is fair. If one is willing, as de Finetti is, to relax countable additivity in response to these considerations, then one can adopt a merely finitely additive probability that assigns each ticket in the integer lottery probability 0 , thus reflecting the opinion that the lottery is fair. ${ }^{10}$

There are various ways to counter de Finetti's argument. One could contest its conclusion - that violations of countable additivity are permissible-by denying the premise that it is sometimes reasonable to believe that a lottery on the integers is fair. Or, one could admit that de Finetti's argument counts as a consideration against mandating countable additivity but claim that this consideration is outweighed by arguments that support mandatory countable additivity. For example, one could appeal to extended Dutch book arguments based on countable collections of bets or to countable additivity's theoretical fruitfulness. Rejoinders to these reservations about de Finetti's position have been voiced a number of times in the literature (e.g., Howson 2014; Kelly 1996).

Our aim in this section is not to adjudicate debates about the integer lottery. We have rehearsed de Finetti's argument in order to show that the best version of the Orgulity Argument has an analogous structure. As we will explain at greater length in the next section, according to proponents of the Orgulity Argument, Bayesianism forbids an opinion that is reasonable, at least in some circumstances, namely, having less than full confidence in converging to the truth. Later in the paper (Section 6), we will push the analogy further when we turn to evaluating a particular reply to the Orgulity Argument based on finitely additive probability.

\section{A Modest Orgulity Argument}

As we have just explained, the inconsistency of countable additivity with a fair lottery on the integers is taken by some as grounds to reject countable additivity as a requirement of rationality. Similarly, we suggest, the correct version of the Orgulity Argument sees the inconsistency of Bayesianism with a "reasonable epistemological modesty" as grounds to reject Bayesianism. We call this version of the argument modest because it relies on a premise that is strictly weaker than the topological conditions that appear in Cisewski et al.'s version.

According to the modest Orgulity Argument, a probability $P$ is modest concerning some hypothesis $H \in \mathscr{B}$ if $P$ assigns the failure set for $H$ positive probability. Such modesty is reasonable if the failure set is comeager (topologically "typical"). "Reasonable" here is interpreted as it was above for the de Finetti lottery, that is,

10. For a relatively recent study of uniform distributions on the natural numbers, see Schirokauer and Kadane (2007). 
as synonymous with "permissible" not with "required." Some agent may adopt a modest opinion regarding $H$ without thereby surrendering his rationality. In order to accuse Bayesianism of orgulity, it is enough to think that it should be rationally permissible for an agent to assign positive probability to a comeager failure set. Such modesty need not be required as Cisewski et al.'s first topological condition puts it. The following topological condition underwrites the modest Orgulity Argument.

Topological Condition \# 3 It is permissible (in some circumstances) to assign positive probability to a comeager failure set.

What is desired, on our reading of Belot, is the existence of some probability $P$ on $(\mathcal{C}, \mathscr{B})$ that assigns a comeager failure set positive probability. Some such $P$ should be rationally permissible. In other words, for some $H$, among the rational probability measures for which the failure set for $H$ is comeager, there should be some $P$ such that

$$
P\left(\left\{\omega: \mathbf{1}_{H}(\omega) \neq \lim _{n \rightarrow \infty} P\left(H \mid \omega^{n}\right)\right\}\right)>0 .
$$

But Bayesianism forbids this, by (1). There is no such probability. So Bayesianism, the argument concludes, should be rejected..$^{11}$

On our view, attacks on Topological Conditions \#1 and \#2 are analogous to attacks on mandatory violations of countable additivity. They do not bear on the modest Orgulity Argument, just as attacks on the position that mere finite additivity is mandatory do not bear on de Finetti's position as we presented it in the last section. Propounders of the Orgulity Argument, as we understand them, are pleading for less stringent epistemological demands, not proposing new ones. We find that this modest version of the Orgulity Argument, even if ultimately wrong, is more compelling than the version that forbids assigning probability 1 to meager sets. It is the modest Orgulity Argument that should be the focus of the debate.

\section{More on Comeager Failure Sets and Finite Additivity}

Having identified the best version of the Orgulity argument, we are now in a position to respond to it. Here, the analogy to de Finetti's criticism of countable

11. Some seem to endorse something akin to our reading of the argument. For example, regarding the assignment of positive probability to a comeager failure set, Elga asks, "Is such humility rationally permissible? According to Gordon Belot's orgulity argument: the answer is yes, but long-run convergence-to-the-truth theorems force Bayesians to answer no" 2016 305, emphasis ours). 
additivity shows itself to be doubly apt. Just as in the integer lottery case, the epistemic state desired by proponents of the Orgulity Argument is permissible if countable additivity is relaxed (Cisewski et al. 2018; Elga 2016). Elga shows that there exists a merely finitely additive probability measure that assigns positive probability to a comeager failure set. ${ }^{12}$ This probability assignment exhibits the sort of epistemological modesty that Belot thinks is reasonable. While a Bayesianism that assumes countable additivity is inconsistent with Topological Condition \#3, Elga's reply shows that finitely additive Bayesianism is not, just as some finitely additive probabilities are consistent with the judgment that the integer lottery is fair.

It seems the relevance of Elga's point has been misunderstood along the same lines as Belot's original argument has. For example, Pomatto and Sandroni claim that concerns about Bayesian orgulity extend to merely finitely additive probabilities because some merely finitely additive probabilities converge to the truth in the context of a certain "hard" kind of inference problem (2018: 15). By our lights, the important point in the context of Pomatto and Sandroni's framework is that, unlike countably additive probabilities, not all merely finitely additive probabilities are fully confident in solving the problem.

Cisewski et al. find Elga's reply unsatisfactory for similar reasons. They complain that his example shows modesty about only one event. They write, "Hence, though Elga argues that $P$ is modest with respect to one limiting frequency hypothesis, according to Condition $\#_{1} P$ is immodest for a different but related hypothesis about existence of well-defined limiting frequencies" (Cisewski et al. 2018: 61).

In view of our articulation of the modest Orgulity Argument, it is now easy to see that these criticisms are misguided. Recall that, as we have shown above, Topological Condition \#1 does not occur as a premise in the Orgulity Argument. Rather, the main premise of the Orgulity Argument is Topological Condition \#3. With this point firmly in mind it is clear that a reply to the Orgulity Argument based on relaxing countable additivity is legitimate. Since it can be shown that there exist merely finitely additive probabilities that assign positive measure to some comeager failure set, it follows that finitely additive Bayesianism is consistent with Topological Condition \#3. This should go some way towards answering Belot's intended challenge.

While we do not find their criticisms of Elga compelling, Cisewski et al.'s remarks do raise the possibility of strengthening the Orgulity Argument's main

12. Cisewski et al. dispute the mathematical details of the particular example Elga offers, arguing that his proofs involve conditioning on null events. They then offer a slightly modified example, which they claim achieves what Elga intends. But even if a merely finitely additive probability assigns positive probability to some comeager failure set, it may assign probability 0 to some other comeager set. 
premise. A premise intermediate to Belot's and Cisewski et al.'s asks that modesty concerning every comeager failure set be permissible under some circumstances. Although not explicitly endorsed by Belot, we find the following condition worthy of further investigation.

Topological Condition \# 4 It is permissible (in some circumstances) to assign positive probability to every comeager failure set.

Topological Condition \#4 is clearly stronger than Topological Condition \#3, but it is weaker than Topological Condition \#1 on two counts. First, in keeping with the main theme of this paper, it claims that certain probability assignments are permissible. It does not mandate any particular assignments. Second, Topological Condition \#4 applies only to comeager failure sets, not to every comeager set. Only the former are relevant to the Orgulity Argument.

Prompted by Cisewski et al.'s criticism, we might ask, Can Elga's reply be extended by showing that finitely additive Bayesianism is consistent with Topological Condition \#4? In fact it can be, as we show with the following proposition.

Proposition 1. There exist uncountably many finitely additive probabilities that assign positive probability to all their comeager failure sets.

Proof. To see that the proposition holds, note that the collection of comeager subsets of $\mathcal{C}$ forms a filter on the powerset of $\mathcal{C}$. That is, the collection of comeager sets contains $\mathcal{C}$ and is closed under supersets and finite intersections. The filter of comeager subsets of $\mathcal{C}$ can be extended to an ultrafilter $\mathcal{U}$, a filter that contains exactly one of $A$ or $A^{c}$ for every $A \subseteq \mathcal{C}$. The set function $U$ defined on the powerset of $\mathcal{C}$ by $U(A)=1$ if $A \in \mathcal{U}$ and $U(A)=0$ if $A \notin \mathcal{U}$ is a finitely additive probability measure that assigns probability 1 to every comeager subset of $\mathcal{C}$. Hence, the restriction of $U$ to the Borel sigma-algebra $\mathscr{B}$, which we will also denote by $U$, is a finitely additive probability measure that assigns probability 1 to every comeager, Borel-measurable subset of $\mathcal{C}$. A fortiori, $U$ assigns positive probability to all of its comeager failure sets. This establishes that $U$ satisfies Topological Condition \#4. To see that there are uncountably many other such finitely additive probabilities, fix $\lambda \in(0,1)$ and observe that the function $P \mapsto \lambda P+(1-\lambda) U$ injects the space of probability measures on $(\mathcal{C}, \mathscr{B})$ into the space of probability measures that satisfy Topological Condition \#4. This proves the proposition.

We remark that the argument just given actually establishes the following result. For any topological space $X$, and any measurable space $(X, \mathcal{F})$, let $\mathbb{P}$ be the collection of finitely additive probabilities $P$ on $(X, \mathcal{F})$ satisfying: $P(A)>0$ for every measurable, comeager subset of $X$. Then the cardinality of $\mathbb{P}$ is equal to the cardinality of the space of all probabilities on $(X, \mathcal{F})$. 
Although the argument just given shows that finitely additive Bayesianism is consistent with Topological Condition \#4, there is an objection to consider. One might regard the probabilities constructed above as mere mathematical curiosities. It's not clear that they bear any resemblance to the more familiar probability constructions that Belot uses to advance his argument. In addition to answering this objection, the next proposition, as we will see, also improves on Proposition 1. To begin, we must first recall how probabilities are typically constructed in the countably additive setting. The standard approach is to first define $P$ on a collection of "simple" or "low-complexity" subsets. One then appeals to an extension theorem to establish that $P$ extends to the entire sigma-algebra generated by the simple subsets. In our setting, the simple subsets are all those of the form $\omega^{n}$, as well as finite unions of these sets. This collection forms an algebra, which we denote by $\mathcal{F}$, and $\mathcal{F}$ generates $\mathscr{B}$. For example, to construct the fair coin measure that was mentioned before, one first defines $P$ by $P\left(\omega^{n}\right)=2^{-n}$ and then shows that $P$ is countably additive on $\mathcal{F}$. By the Carathédory extension theorem, $P$ extends to a unique countably additive probability whose domain is all of $\mathscr{B}$.

This is how more or less every familiar and interesting countably additive measure on $(\mathcal{C}, \mathscr{B})$ is constructed. ${ }^{13}$ The next result shows that any $P$ defined on $\mathcal{F}$ can be extended to a finitely additive probability that is consistent with Topological Condition \#4. This means that for every countably additive probability, there exists a finitely additive probability that "resembles" it in the sense that the two agree on $\mathcal{F}$, and the finitely additive probability is modest in the strong sense of condition \#4.

Proposition 2. Let $P$ be a finitely additive probability with domain $\mathcal{F}$. Let $\mathcal{G}$ be any algebra containing $\mathcal{F}$. There exists an extension $P^{\prime}$ of $P$ to $\mathcal{G}$ such that $P^{\prime}(C)=1$ for every comeager subset $\mathrm{C}$ of $\mathcal{C}$ in $\mathcal{G} .^{14}$

Proof. The proof follows closely the proof of Theorem 4 in Pomatto et al. (2014). Let $\mathcal{M}$ be the collection of meager subsets of $\mathcal{C}$, and let

$$
\mathcal{A}=\left\{(F \cap C) \cup M: F \in \mathcal{F}, C^{c} \in \mathcal{M} \cap \mathcal{G}, M \in \mathcal{M} \cap \mathcal{G}\right\} .
$$

Observe that $\mathcal{F} \subseteq \mathcal{A} \subseteq \mathcal{G}$. One can now verify that $\mathcal{A}$ is an algebra, and that the function $P^{\prime}$ defined on $\mathcal{A}$ by

$$
P^{\prime}((F \cap C) \cup M)=P(F)
$$

13. There are ways to define $P$ directly, without appeal to extension theorems, for example, by letting $P$ be point mass concentrated on some $\omega \in \mathcal{C}$. tion 1

14. Thanks to Belot for pointing out in conversation that Proposition 2 strengthens Proposi- 
is a well-defined, finitely additive probability measure (see the reference above for details). By construction, with $F=\varnothing, P^{\prime}(M)=0$ for every meager subset $M$ in $\mathcal{G}$. With a harmless abuse of notation, $P^{\prime}$ now extends to $\mathcal{G}$, and the result is proved.

Our propositions show that replies to the Orgulity Argument based on finitely additive probability are robust. By relaxing countable additivity, various notions of modesty akin to the one in which Belot is interested are easily accommodated. ${ }^{15}$

As with de Finetti's lottery argument, there are responses to the Orgulity Argument besides relaxing countable additivity. One could deny the basic assumption that topology is of any relevance to epistemology, for example (Huttegger $2015 b) .{ }^{16}$ In fact, we are quite sympathetic to this line of response. In order for the Orgulity Argument to be truly convincing, an explanation of when and why confidence should coincide with topological typicality is needed. An effort to fill this gap would be an interesting line of research. However, as a reply to the Orgulity Argument, appealing to finitely additive probabilities has the rhetorical advantage of allowing us to grant both the general relevance of topology to epistemology and the various specific topological conditions for the sake of the argument. Even with these assumptions granted for the sake of the argument, finitely additive Bayesianism need not be abandoned. Alternatively, one may find the assumptions of the Orgulity Argument plausible or genuinely compelling. In that case, Elga's point and our propositions contribute to the case against mandating countable additivity.

\section{Conclusion}

The modest Orgulity Argument takes as a premise that it is permissible, at least sometimes, to assign probability greater than 0 to a comeager failure set. It does not appeal to a universal injunction against assigning probability 1 to meager sets à la Topological Condition \#1. To do so would be analogous to claiming that countably additive probabilities are rationally forbidden. We hope to have helped to focus future debate on the modest version of the Orgulity Argument that we have articulated here.

Following Elga, we have also defended a reply to the modest Orgulity Argument that appeals to finitely additive probability. We have argued that this reply is robust by considering topological conditions that are stronger than those

15. One can glean from the above arguments that even Cisewski et al.'s demanding conditions are satisfiable by moving to finitely additive probabilities and restricting the permissible probabilities to those mentioned in the propositions.

16. Pollard observes, "Many probabilists seem to regard topology as completely extraneous to any discussion of conditioning, or even to any discussion of abstract probability theory" 2002 117). 
that Belot appeals to but weaker and more plausible than those of Cisewski et al. In particular, Topological Condition \#4 claims that it is permissible in some circumstances to assign positive probability to all comeager failure sets. We have shown that finitely additive probabilities can meet the even more demanding challenges raised by this new condition. There are uncountably many finitely additive probabilities on $(\mathcal{C}, \mathscr{B})$ that assign positive probability to all their comeager failure sets. Just as in de Finetti's lottery, finitely additive probability permits agents to adopt seemingly reasonable states of probability judgment that are ruled out by countable additivity.

\section{References}

Belot, Gordon (2013). Bayesian Orgulity. Philosophy of Science, 8o(4), 483-503.

Belot, Gordon (2017). Objectivity and Bias. Mind, 126(503), 655-695.

Billingsley, Patrick (2008). Probability and Measure. John Wiley \& Sons.

Cisewski, Jessi, Joseph B. Kadane, Mark J. Schervish, Teddy Seidenfeld, and

Rafael Stern (2018). Standards for Modest Bayesian Credences. Philosophy of Science, 85(1), 53-78.

de Finetti, Bruno (1974). Theory of Probability (Vol. 1). John Wiley \& Sons.

Elga, Adam (2016). Bayesian Humility. Philosophy of Science, 83(3), 305-323.

Howson, Colin (2014). Finite Additivity, another Lottery Paradox and Conditionalisation. Synthese, 191(5), 989-1012.

Huttegger, Simon M. (2015a). Bayesian Convergence to the Truth and the Metaphysics of Possible Worlds. Philosophy of Science, 82(4), 587-601.

Huttegger, Simon M. (2015b). Merging of Opinions and Probability Kinematics. The Review of Symbolic Logic, 8(04), 611-648.

Kelly, Kevin T. (1996). The Logic of Reliable Inquiry. Oxford University Press.

Oxtoby, John C. (2013). Measure and Category: A Survey of the Analogies Between Topological and Measure Spaces (Vol. 2). Springer Science \& Business Media.

Pollard, David (2002). A User's Guide to Measure Theoretic Probability. Cambridge University Press.

Pomatto, Luciano and Alvaro Sandroni (2018). An Axiomatic Theory of Inductive Inference. Philosophy of Science, 85(2), 293-315.

Pomatto, Luciano, Nabil Al-Najjar, and Alvaro Sandroni (2014). Merging and Testing Opinions. The Annals of Statistics, 42(3), 1003-1028.

Schirokauer, Oliver and Joseph B. Kadane (2007). Uniform Distributions on the Natural Numbers. Journal of Theoretical Probability, 20(3), 429-441.

Weatherson, Brian (2015). For Bayesians, Rational Modesty Requires Imprecision. Ergo, 2(20), (529-545). 